\title{
Rats' responses to molar and local schedule constraints
}

\author{
JAMES ALLISON, ALISON BUXTON, and KEVIN E. MOORE \\ Indiana University, Bloomington, Indiana
}

\begin{abstract}
Three experiments explored responses to molar and local schedule constraints. Thirsty rats pressed a lever for access to a water spout. In Experiment 1, response totals were unaffected by two local schedule characteristics-the variability of the instrumental requirement and the variability of the magnitude of contingent reward. Experiment 2 manipulated the correlation between the instrumental requirement and the magnitude of reward. This correlation did not affect the behavioral price ratio (presses per lick) at a molar level. At a local level, the positive correlation created a lower mean lick price than did the negative correlation. The rats licked more, and licked less efficiently, under the positive correlation than under the negative correlation. Experiment 3 compared two ways of manipulating the molar presses/lick ratio: The instrumental (contingent) series varied the instrumental (contingent) requirement, but held the other requirement constant. As the ratio increased, total leverpresses increased, and total licks decreased linearly; the two series did not differ significantly. At higher lick prices, the rats licked more efficiently and made more extra licks at the spout as it closed. The results help delimit the applicability of molar models of the organism's response to schedule constraints.
\end{abstract}

Numerous models of behavior under schedule constraints, such as response deprivation (Allison \& Timberlake, 1974; Timberlake \& Allison, 1974), minimum deviation (Staddon, 1979), and conservation (Allison, 1976; Allison, Miller, \& Wozny, 1979), focus on the schedule's molar features, rather than its local features. Such models assume that the individual responds mainly to overall schedule constraints. For example, they would predict similar response totals for rats trained under two different fixed-ratio schedules, one that required 10 leverpresses for 10 licks at a waterspout, and another that required 20 presses for 20 licks. Despite the fact that the local instrumental and contingent requirements differ by a factor of 2 , these models would predict similar response totals, because both schedules employ the same molar ratio--one leverpress per lick.

Allison and Timberlake (1974) reported evidence in support of the predicted similarity. They tested rats with four schedules that required 100 instrumental licks of a sweeter saccharin solution for 10 contingent licks of the other saccharin solution, 200 for 20,300 for 30 , and 400 for 40 . The rats performed essentially the same number of responses under all four schedules-about 1,000 licks at the sweeter solution, 100 at the other. However, rats have proved sensitive to local schedule constraints under special conditions, including an unusually great travel distance between the instrumental lever and the contingent waterspout. Allison et al. (1979) imposed reciprocal contingency schedules that required $5 \mathrm{sec}$ of leverholding for 5 sec of drinking, 10 for 10 , and 20 for 20 -schedules with different local requirements, but equal molar ratios.

Reprint requests should be sent to James Allison, Department of Psychology, Indiana University, Bloomington, IN 47405.
When the travel distance between the lever and the waterspout was short, the three schedules resulted in essentially the same behavioral totals-about $635 \mathrm{sec}$ of holding and $635 \mathrm{sec}$ of drinking under each schedule. But when the travel distance was long, the behavioral totals decreased monotonically as the schedule requirements fell from $20 / 20$ to $10 / 10$ and 5/5. This empirical exception suggests that long trips may increase the rat's sensitivity to local schedule constraints.

In the present experiments, we investigated the rat's response to local variations among schedules with the same molar constraints. Our purpose was to explore further the molar models' limits of applicability. One experiment manipulated the variability of the instrumental and contingent requirements while holding constant the overall instrumental/contingent ratio. Another studied the effects of local positive and negative correlations between the instrumental and contingent requirements. The third experiment compared two ways of manipulating the instrumental/contingent ratio: An instrumental series varied the number of leverpresses required for a constant number of licks; a contingent series varied the number of licks allowed for a constant number of leverpresses.

\section{EXPERIMENT 1}

In this experiment we compared four types of reciprocal contingency schedule in terms of their effects on performance during training and extinction. We created the four by either varying or holding fixed the instrumental leverpress requirement and the magnitude of the contingent water reward. The four schedules that resulted from these factorial combinations had the same average instrumental and contingent requirements. 
One of our four types, the fixed-fixed, is a conventional fixed-ratio schedule. Under this type of schedule the instrumental requirement is fixed, and so is the contingent reward. An example would be a schedule that always requires $\mathbf{1 0}$ leverpresses for access to water, and always allows 20 licks at the waterspout per access. A second type, variable-fixed, is a conventional variable-ratio schedule. Under this type of schedule the instrumental requirement varies, but the magnitude of reward is fixed. An example would be a schedule that selects at random 5,10 , or 15 presses as the current requirement for access to the spout, and allows 20 licks during each access.

More unusual are the third and fourth types. The fixedvariable schedule combines a fixed instrumental requirement with a variable magnitude of reward. An example would be a schedule that requires 10 presses for access, and allows at random 10,20 , or 30 licks. The variablevariable type varies both the instrumental requirement and the magnitude of reward. An example would be a schedule that selects at random 5,10 , or 15 presses as the instrumental requirement and allows at random 10,20 , or 30 licks.

The four examples specified above are the schedules used in Experiment 1 . Note that although the local instrumental and contingent requirements differ across the schedules, the overall instrumental/contingent ratio is constant at 0.5 presses/lick. Consequently, models that focus on molar schedule constraints would predict similar responses to the four schedules.

\section{Method}

Subjects. Twelve 90-day-old Sprague-Dawley rats, experimentally naive males purchased from Harlan Industries (Indianapolis, IN), served in the experiment.

Apparatus. The rats were tested 3 at a time in three identical chambers controlled by a microcomputer. A hinged Plexiglas door on the front of the chamber exposed a sheet-metal interior measuring $26 \mathrm{~cm}$ wide, $24 \mathrm{~cm}$ deep, and $16 \mathrm{~cm}$ high. In the wall opposite the door was a circular hole fitted with a motorized shutter. The shutter controlled access to a stainless steel drinking spout connected to a graduated water tube. The hole was $2.5 \mathrm{~cm}$ wide, $5 \mathrm{~cm}$ from the left edge of the wall, and $9 \mathrm{~cm}$ above the floor. The spout, recessed $1 \mathrm{~cm}$ beneath the surface of the wall, had a 2-mm aperture. An electronic drinkometer registered each discrete contact between tongue and spout. We recorded volumetric intake to the nearest $0.5 \mathrm{ml}$, corrected for spillage in a catchment.

A food trough $6 \mathrm{~cm}$ above the floor and $13 \mathrm{~cm}$ to the right of the waterspout gave access to about $10 \mathrm{~g}$ of 45 -mg food pellets (BioMix T1300, Bio-Serv, Inc.; Frenchtown, NJ). A retractable lever protruded through an oblong cutout midway between the spout and the trough, $9 \mathrm{~cm}$ above the floor. Each box was illuminated by a small light on the wall $3 \mathrm{~cm}$ above the lever. A $15-\mathrm{W}$ bulb hanging from the ceiling lighted the room that housed the chambers, and a noise generator masked extraneous sounds.

Procedure. Before the first experimental session we tamed the rats and restricted their water intake to the $1-\mathrm{h}$ periods reserved for their test sessions. Food was freely available throughout the experiment in both the home cage and the food trough in the test chamber. The rats always ate during the test session, but never exhausted the trough's supply of pellets. They performed during the first three sessions under a paired baseline condition that allowed free access to both lever and waterspout. Starting with the fourth session, we pretrained them with reciprocal fixed-ratio contingency schedules that required either one or two leverpresses for 20 licks. In accordance with the definition of a reciprocal schedule, upon each completion of the leverpress requirement, the lever retracted and the spout appeared; upon each completion of the lick requirement, the shutter covered the spout and the lever reappeared. We continued pretraining until it appeared that the individual subject had learned the contingency; the number of pretraining sessions ranged from one to four. Immediately after any session in which the rat drank less than one third of the volume consumed during its median paired baseline session, we allowed 15 min of free access to supplemental water in its home cage.

We assigned 3 rats to each of the four schedules by matching four groups in terms of the median number of licks in the paired baseline sessions. The fixed-fixed schedule required 10 leverpresses for 20 licks. The variable-fixed schedule required 5,10 , or 15 presses for 20 licks. The fixed-variable schedule required 10 presses for 10,20 , or 30 licks. The variable-variable schedule required 5 , 10 , or 15 presses for 10,20 , or 30 licks. Within each block of three accesses to the spout, the computer selected the variable requirements at random without replacement; thus, there should have been no correlation between the current press (lick) requirement and the next lick (press) requirement.

We trained each rat under its assigned schedule for at least four sessions. The number of training sessions ranged from four to seven. When leverpress totals were nonmonotonic over the three most recent sessions, we ended the training phase. All but 1 of the animals were tested the following day under an experimental extinction condition with the lever alone present thoughout the session. Because it appeared to be ill on the appointed day, 1 rat in the variablefixed condition was not tested under the extinction condition.

Our session totals included leverpresses, licks, and volumetric intake. We also recorded session means for two latency measures: leverpress latency, the time from each presentation of the lever to the first leverpress; and lick latency, the time from each presentation of the spout to the first lick. In addition, we recorded session means for lever interresponse time and lick interresponse time, the inverse of which is responses per second. More specifically, for each lever (spout) presentation, mean interresponse time consisted of the mean time between successive presses (licks) in the presence of the lever (spout).

\section{Results and Discussion}

We conducted several two-way analyses of variance with the variability of the instrumental and contingent requirements as the two between-subjects factors. The training data were measures obtained in the representative training session, defined as that one of the last three sessions that contained the individual's median leverpress total. The extinction data consisted of the number of leverpresses in the extinction session.

In agreement with molar models of performance under schedule constraints, the analyses of training data revealed no significant main effects or interactions with respect to total leverpresses or licks. They also revealed no significant main effects or interactions with respect to volumetric intake, leverpress latency, lick latency, or leverpress rate. Note again that the local variability of instrumental and contingent requirements had no significant effects on total leverpresses or total licks in the representative training session. The latter findings differed from those of Ferster and Skinner (1957), who reported that pigeons responded more on variable- than on fixed-ratio 
schedules. Figure 1 shows the group means for total leverpresses during training and extinction (top panel), licks (middle panel), and volumetric intake (bottom panel). The group means for leverpress latencies and lick latencies appear in the top panel of Figure 2.

We found significantly more leverpresses in training than in extinction $[t(10)=14.55, p<.001]$. However, we found no difference in extinction between rats trained with fixed and those trained with variable instrumental requirements. The latter finding contradicts the widespread impression that if the schedules have the same instrumental/contingent ratio, animals trained on variable-
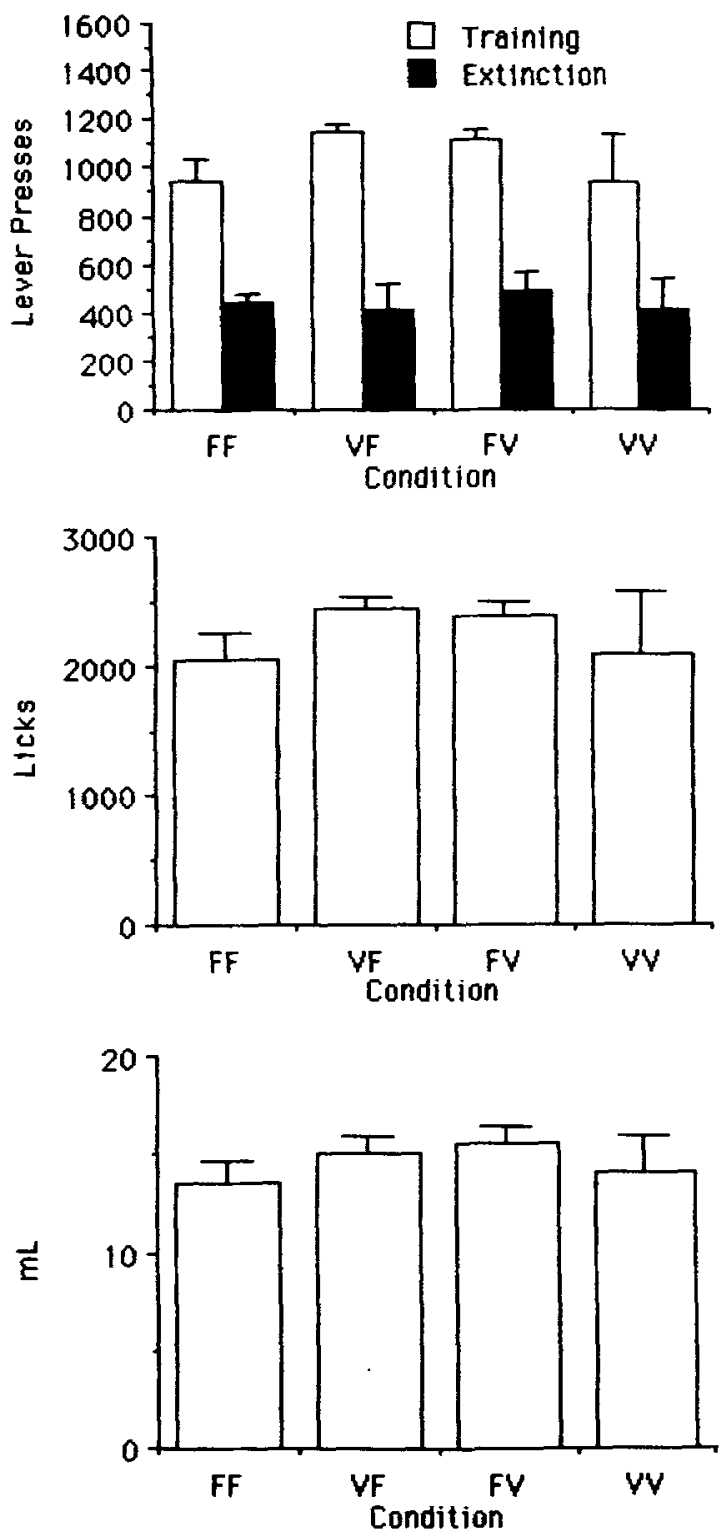

Figure 1. Leverpresses, licks, and volumetric intake as functions of the variability of the instrumental and contingent requirements (group means and standard errors); the schedules required a fixed or variable number of leverpresses for a fixed number of licks (FF and VF) or for a variable number of licks (FV and VV).
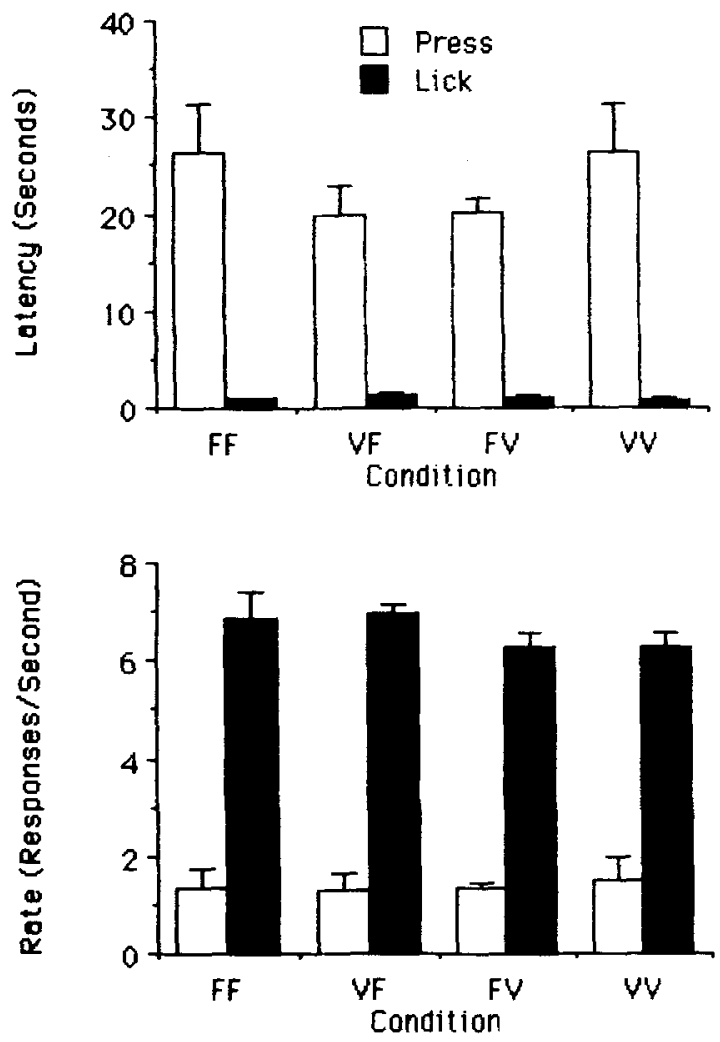

Figure 2. Response latencies and rates during training as functions of the variability of the instrumental and contingent requirements (group means and standard errors); the schedules required a fixed or variable number of leverpresses for a fixed number of licks (FF and VF) or for a variable number of licks (FV and VV).

ratio schedules respond more during experimental extinction than do those trained on fixed-ratio schedules (e.g., Gleitman, 1983, p. 88). Of course, our rats might have responded more in both training and extinction had they been trained with a wider range of variable requirements.

Unlike the other dependent measures, lick rates revealed a significant main effect of variability in the magnitude of reward. Specifically, rats trained on schedules with fixed magnitudes of reward showed significantly higher lick rates $(6.9$ licks $/ \mathrm{sec})$ than those trained with variable magnitudes of reward $(6.3$ licks $/ \mathrm{sec})[F(1,8)=5.79, p<$ $.05]$. Perhaps the greater uncertainty about the closing of the spout shutter induced the rats trained with a variable magnitude of reward to lick more haltingly than those trained with a fixed magnitude of reward. Group means for response rates appear in Figure 2 (bottom panel).

So far, our analysis of local features has focused on the variability of the instrumental and contingent requirements. Another feature worth study is the statistical correlation between those requirements. In each of the four schedules used in Experiment 1, there was no correlation between the number of instrumental leverpresses required for the next access to the spout, and the number of licks allowed during that access. Three schedules guaranteed 
a correlation of zero by using a fixed instrumental requirement and/or a fixed contingent requirement. Under the fourth schedule, the correlation should have approached zero: In implementing the variable-variable schedule, the computer selected each of the two variable requirements independently of the other. Inspection of $3 \times 3$ contingency tables recorded by the computer during the course of all training sessions showed that the correlation did approach zero: For each of the three press requirements, the three lick requirements occurred about equally often. But most important, we can easily imagine other variable-variable schedules in which the correlation would be positive or negative rather than zero. In Experiment 2, we investigated the behavioral effects of these local correlations between the instrumental and contingent requirements.

\section{EXPERIMENT 2}

This experiment introduced two modifications of the variable-variable schedule used in Experiment 1. In the new positive (negative) schedule, completion of the 5, 10, or 15 leverpresses required for access to the spout allowed 10,20 , or $30(30,20$, or 10$)$ licks, respectively, during that access. Thus, under the positive (negative) schedule, $r=+1(-1)$ : Completion of a relatively large instrumental requirement always led to a relatively large (small) magnitude of reward. Under the uncorrelated schedule used in Experiment 1, $r=0$ : There was no consistent relation between the size of the press requirement and that of the lick requirement.

Molar behavioral models imply that the total amount of responding under schedule constraints should not depend on the local correlation between instrumental and contingent requirements. To understand this implication, note that the molar expected value of the instrumental/ contingent ratio for the session as a whole was precisely the same for the positive schedule, the negative schedule, and the uncorrelated schedule used in Experiment 1 . That molar expected value was $(1 / 3)(5+10+15) /$ $(1 / 3)(10+20+30)$, or 0.5 presses/lick, for each of those three schedules. Accordingly, molar models would predict similar response totals for rats tested under the positive schedule and rats tested under the negative schedule.

Alternatively, if the rat responds to local values of the instrumental/contingent ratio, we have reason to expect that the positive and negative schedules might generate different response totals. To understand the reason, note that the three local values of the instrumental/contingent ratio differ between the two schedules. For the positive schedule, the three local values are $5 / 10,10 / 20$, and $15 / 30-0.5,0.5$, and 0.5 presses/lick. Their expected value, $(1 / 3)(0.5+0.5+0.5)$, is 0.5 presses/lick-the same as the molar expected value. But for the negative schedule, the three local values are $5 / 30,10 / 20$, and 15/10 $0.17,0.5$, and 1.5 presses/lick. Their expected value is $(1 / 3)(0.17+0.5+1.5)$, or 0.72 presses/lick $-44 \%$ higher than the molar expected value, and of course higher than the local expected value for the positive schedule.
Thus, according to local calculations of expected value, water comes at a higher behavioral price (presses per lick) in the negative schedule than in the positive schedule. There is abundant evidence that rats pressing levers for water and other such economic goods behave in accordance with the demand law, consuming less of a particular good as its unit price rises (Allison, 1983). Accordingly, if the rat responds to local values of the instrumental/contingent ratio, we should find more total leverpresses and licks under the positive schedule than under the negative schedule: By the local calculations, water is simply cheaper under the positive schedule than under the negative schedule.

\section{Method}

Subjects and Apparatus. Eight rats from Experiment 1 performed in the same chambers used in that experiment. From three of the four conditions in Experiment 1, we discarded 1 rat at random from each condition. The variable-variabie condition included 1 rat with an unusually low level of performance; accordingly, we discarded that rat.

Procedure. We restricted the rats' water intake to the 1-h periods reserved for their test sessions. They had free access to food at all times.

To equate their preexperimental treatment, we pretrained the rats for three sessions under the variable-variable schedule used in Experiment 1 , with no correlation between the requirements. During the next three sessions, the computer generated either a perfect positive correlation $(r=1)$ or a perfect negative correlation $(r=-1)$ between the number of leverpresses required for the next access to water $(5,10$, or 15$)$ and the number of licks required for the next presentation of the lever $(10,20$, or 30). As in Experiment 1, the computer selected the requirements at random without replacement within each block of three accesses. We assigned 4 rats to the positive schedule and 4 to the negative by matching the two groups as closely as we could in terms of the median number of leverpresses during the pretraining sessions under the uncorrelated schedule.

After each session we recorded the same measures recorded in Experiment 1 and gave supplemental water in the home cage in accordance with the same guidelines.

\section{Results and Discussion}

For every dependent variable reported here, we used analysis of covariance to equate the two groups statistically in terms of their performance under the uncorrelated schedule. Contrary to molar expectation, the local correlation between the instrumental requirement and the magnitude of contingent reward had a significant effect on total leverpresses and licks. Specifically, the rats in the positive correlation condition performed significantly more leverpresses than did those in the negative condition $[F(1,5)=10.52, p<.05]$; the adjusted means were 1,219 and 974 . The positive group also made significantly more licks than did the negative group $[F(1,5)=14.34$, $p<.05$ ]; the adjusted means were 2,673 and 2,088 . These differences cannot be attributed to any appreciable difference in the molar ratio actually experienced by the two groups -0.46 presses/lick for the positive group, on average, and 0.47 for the negative. Group means for the leverpress and lick totals appear in the top panels of 
Figure 3. The differences shown there are consistent with the hypothesis that the rats were sensitive to the local press/lick ratio-that the expected value of that ratio, and thus the local behavioral price of water, was higher under the negative than under the positive schedule.

There were no significant differences in terms of the latency of the first leverpress, the latency of the first lick, or the rate of subsequent responses. However, other analyses revealed an unexpected "microbehavioral" adjustment of some importance. Specifically, rats in the negative correlation condition licked more efficiently than did those in the positive condition. Still more specifically, the negative group made significantly fewer licks per milliliter than did the positive group $[F(1,5)=8.39, p<.05]$; the adjusted means were 142 and 172 licks/ml. Thanks to their superior lick efficiency, the negative group did not differ significantly from the positive group in terms of total volumetric intake (see the bottom panels in Figure 3). Our third experiment gave more direct evidence on the possible functional relation between the behavioral price of water and lick efficiency.

\section{EXPERIMENT 3}

In this experiment, we compared alternative ways of varying the behavioral price of a commodity. The mar-
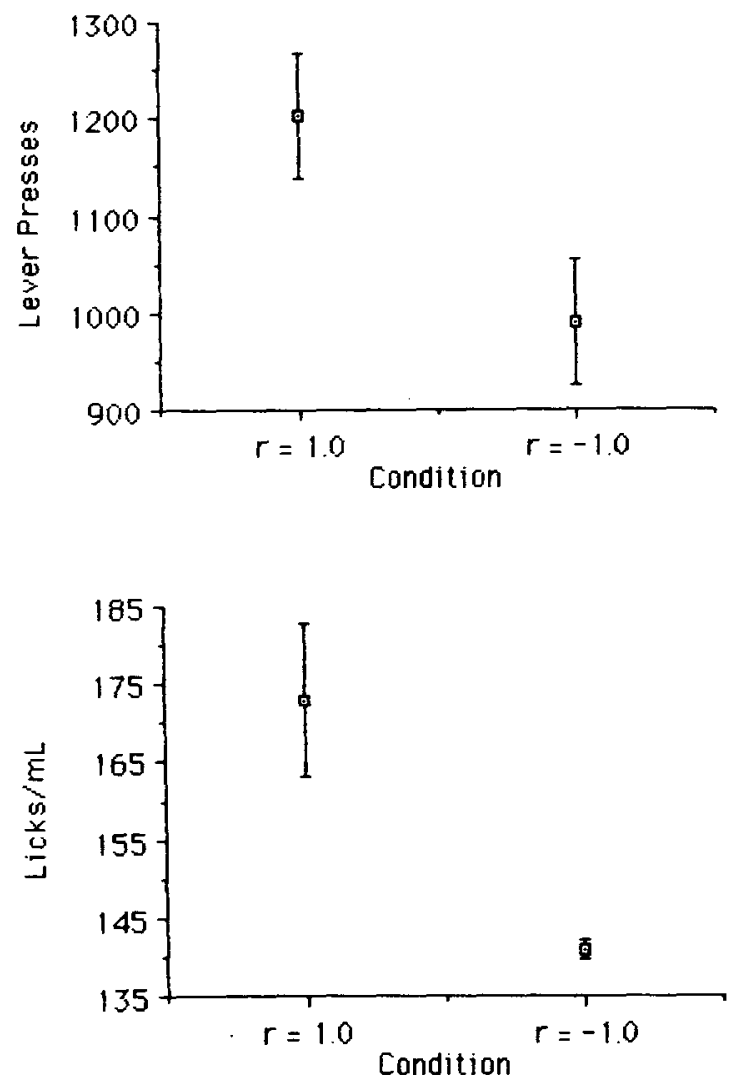

keter may manipulate the unit price of a good by altering either the price of the package or the package size. For example, to increase the unit price of candy (dollars per gram) one might charge either a higher price for the same candy bar or the same price for a smaller bar. In the present experiment we manipulated the unit price of water (the number of leverpresses per lick) in two ways. Four simple fixed-ratio schedules formed an instrumental series analogous to four different prices for the same bar. They required $5,20,80$, or 120 leverpresses for access to water, and 24 licks for the next presentation of the lever. The corresponding nominal unit prices were therefore approximately $0.2,0.8,3.3$, and 5.0 presses/lick. Four other simple fixed-ratio schedules formed a contingent series analogous to the same price for four different bar sizes. They required 20 leverpresses for access to water and $96,24,6$, or 4 licks for the next presentation of the lever. Accordingly, their corresponding unit prices were also $0.2,0.8,3.3$, and 5.0 presses/lick.

If their response is based on molar constraints, rats should respond similarly under schedules that establish the same behavioral price by means of different instrumental and contingent requirements. Because the instrumental and contingent series charge the same unit prices by having the same instrumental/contingent ratios, they should result in similar behavioral functions. Allison et al.
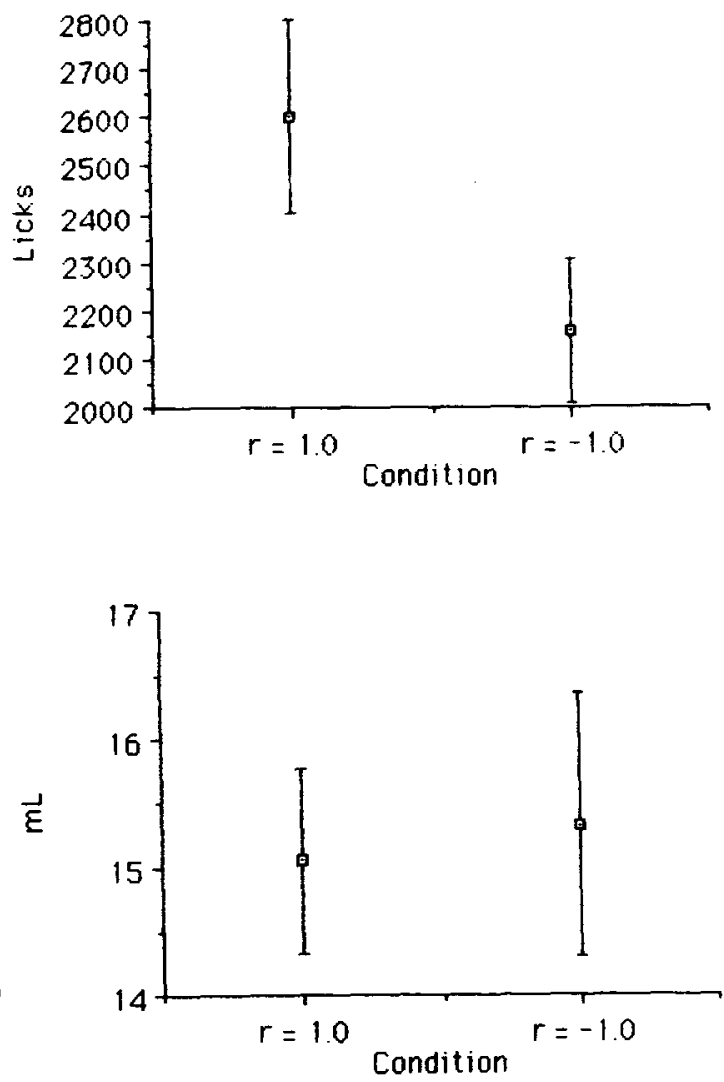

Figure 3. Leverpresses, licks, licks per milliliter, and volumetric intake as functions of the correlation between the instrumental and contingent requirements (group means and standard errors). 
(1979) tested this prediction by manipulating either the instrumental requirement or the contingent requirement across schedules. As predicted, the results showed similar behavioral totals under two pairs of schedules that had different instrumental and contingent requirements, but equal instrumental/contingent ratios. The present study extended the earlier work by testing more schedules, four pairs rather than two, over a much wider range of prices. Because of the unexpected microbehavioral adjustments revealed by Experiment 2, we also examined the relation between price and lick efficiency.

\section{Method}

Subjects and Apparatus. Eight of the rats from Experiment 1, selected at random, performed in the same apparatus used in Experiments 1 and 2 .

Procedure. Except as noted, we followed the same general procedures used in the two preceding experiments. Before we started the series of training sessions, we pretrained each rat under a simple fixed-ratio schedule that required 5 leverpresses for access to water and 24 licks for the next presentation of the lever. Six rats received one pretraining session, and 2 received two sessions.

From each test session to the next we manipulated the press/lick ratio by varying either the instrumental leverpress requirement, in the instrumental series, or the contingent lick requirement, in the contingent series. The rats experienced each schedule twice, first in order of increasing instrumental and contingent requirements, and then in order of decreasing contingent and instrumental requirements.

\section{Results and Discussion}

We applied three-way analyses of variance with repeated measures on each factor, using logarithmic transformations when the data appeared positively skewed. The three factors were the nominal instrumental/contingent ratio, series, and order. Recall that we controlled for order effects by testing the rats on the schedules first in an ascending order and then in a descending order.

Figure 4 presents the group means for total leverpresses and total licks in the instrumental series and in the contingent series. Four schedule constraint lines representing the nominal instrumental/contingent ratios--approximately $0.2,0.8,3.3$, and 5.0 presses/lick-radiate from the origin. Our statistical analyses of the data summarized in Figure 4 showed that increases in price (presses per lick) were accompanied by a significant increase in total leverpresses $[F(3,21)=244.00, p<.001]$ and a significant decrease in total licks $[F(3,21)=172.53, p<.001]$. Volumetric intake showed the same functional relations as did licks. In agreement with molar expectations, there was no significant difference between the instrumental and contingent series.

Visual inspection of the data presented in Figure 4 prompted our use of linear regression analysis to compare the behavioral functions generated in the instrumental and contingent series. Both functions appeared to be linear. Each one revealed a strong negative linear relation between total leverpresses and total licks $-r^{2}=.95$ in the instrumental series, .96 in the contingent series. In the instrumental series, $r^{2}$ ranged from .58 to .99

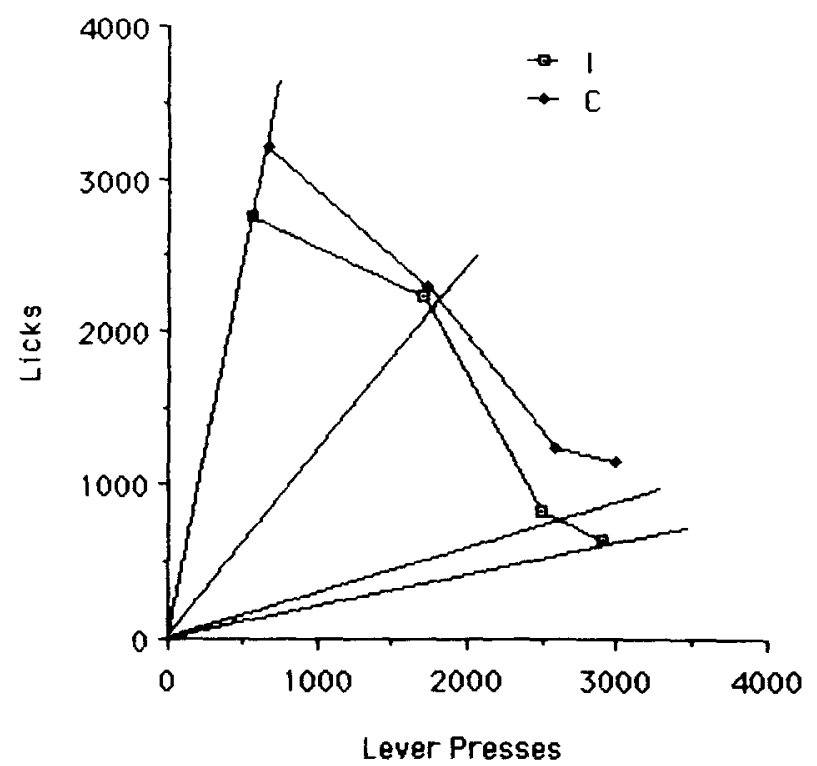

Figure 4. Licks as a function of leverpresses (group means) in the two series, instrumental (I) and contingent (C); lines radiating from the origin represent nominal schedule constraints.

among the 8 individual rats, and the range was from .43 to .99 in the contingent series; each of the 16 correlations was negative. To determine whether the two linear functions differed significantly from each other, we fitted two least squares regression lines to each individual's data, and compared the instrumental-series constants with the contingent-series constants. We found no significant difference between series in terms of the $y$-intercepts or slopes $[t(7)<1.00$ for both the intercept constant and the slope constant]. Thus, our results indicated that the subjects responded similarly in both series, consistent with the results of Allison et al. (1979). The regression line fitted to the means of the two series had an intercept of approximately 3,670 and a slope of $-0.96\left(r^{2}=.96\right)$.

Although we found no significant series effect on overall responding, we found a highly significant effect on the real price of the lick, defined as total leverpresses/total licks actually performed under a particular schedule. Analyses of interactions and simple effects showed that the rats paid significantly less in the contingent than in the instrumental series under the two schedules with the highest nominal prices $(p<.001)$ (see Figure 4). Further analyses, detailed below, identified two separate underlying mechanisms, each having to do with the rat's exploitation of a particular operating characteristic of our apparatus.

In this particular apparatus it takes about $0.5 \mathrm{sec}$ for the shutter to cover the spout completely after the rat has satisfied the nominal lick requirement. During that time the rat may perform a few extra licks beyond the nominal requirement, and so pay less than the nominal price. This overrunning of the nominal requirement accounted for the series effect upon the real price of the lick. The 
first of the two mechanisms had to do with the size of the press requirement. At the two highest nominal prices, 3.3 and 5.0 presses/lick, the requirement was smaller in the contingent series ( 20 presses) than in the corresponding instrumental series ( 80 or 120 presses). Accordingly, at those two prices, comparable leverpress totals brought significantly more accesses to the spout under the contingent-series schedule, more chances to overrun the nominal lick requirement, and a lower price. For example, under the 120 presses/24 licks schedule, assuming 2 extra licks per access, 120 presses would allow 26 licks: $1(120) / 1(24+2)=120 / 26=4.6$ presses $/$ lick. In contrast, under the $20 / 4$ schedule, 120 presses would allow 36 licks: $6(20) / 6(4+2)=120 / 36=3.3$ presses $/$ lick.

The second mechanism we identified involved a more active and more subtle adaptation. Specifically, the rats made significantly more extra licks per opportunity in the contingent series than in the instrumental series under the schedules that charged the two highest prices. This interaction between series and the nominal price imposed by the schedule is evident in Figure 5. Analysis of the simple effects displayed in Figure 5 revealed still another significant adaptation: For both series, the number of extra licks per opportunity increased significantly as the nominal price increased $[F(3,21)=14.99$ for the instrumental series, 37.20 for the contingent; $p<.001$ in both cases]. In contrast, none of our experimental manipulations affected the number of extra leverpresses per opportunity; the grand mean was about 0.7 .

Our analyses confirmed the unexpected adjustment revealed by Experiment 2 , in which lick efficiency increased with price. Specifically, the rats performed significantly more licks per milliliter at the lowest price ( 0.2 presses/lick) than at the other three prices combined $[F(3,7)=6.59, p<.05]$. Thus, the rats again licked more efficiently as the schedule exacted a higher behavioral price for water.

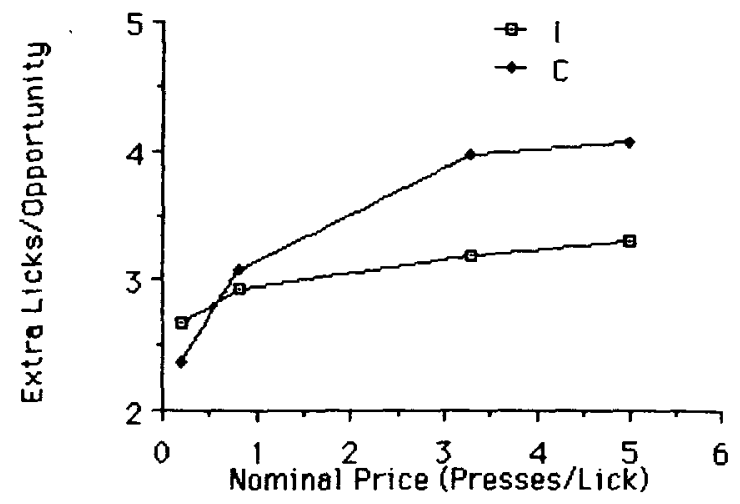

Figure 5. Licks in excess of the nominal lick requirement as a function of the nominal schedule requirements (presses per lick) in the instrumental series (I) and the contingent series (C).

\section{GENERAL DISCUSSION}

In these experiments, we explored the behavioral effects of local and molar schedule constraints. In agreement with molar models, our rats responded mainly to the overall schedule constraints. The rats did, however, respond to one local constraint manipulation.

In Experiment 1, our four schedules had the same overall instrumental/contingent ratio, but differed in the variability of the instrumental and contingent requirements. We found that the variability of the requirements had no significant effect on response totals. In Experiment 3, the rats responded to the molar constraints of the schedule, number of presses per lick: As the ratio increased, total leverpresses increased and total licks decreased. However, the rats proved insensitive to the way in which we manipulated that ratio, giving the same response totals in the instrumental and contingent series.

The response totals in Experiment 2, unlike those in Experiments 1 and 3, revealed a significant effect of a local schedule constraint. Specifically, the rats responded to the local correlation between the instrumental and contingent requirements by pressing and licking more under the positive correlation than under the negative correlation, and licking less efficiently under the positive correlation. These differences seem to identify a new condition under which rats are sensitive to the local instrumental/ contingent ratio. If our interpretation is correct, the rats performed more licks under the positive schedule, and licked less efficiently, because the expected behavioral price of the lick, based on the three local press/lick ratios, was lower under the positive than under the negative schedule.

Alternatively, the correlation between the instrumental requirement and the magnitude of reward may influence behavior through something other than a local price mechanism. Note in this regard that the four uncorrelated schedules used in Experiment 1 imposed two local prices. Under each of the two schedules with a fixed magnitude of reward, the expected local price was 0.5 presses/lick. Under each of the two schedules with a variable magnitude of reward, it was slightly higher$0.61 \mathrm{presses} /$ lick. However, these local price differences produced none of the behavioral differences produced in Experiment 2. Perhaps the price variable had a more powerful influence in Experiment 2 simply because it ranged more widely there, from 0.5 presses/lick to 0.72 presses/lick. Alternatively, the effects of local price may depend in some important way on the correlation between the instrumental requirement and the magnitude of contingent reward. Thus, the price variable may be more effective when the local schedule requirements are correlated than when they are uncorrelated.

Theorists concerned with the response to schedule constraints should also take note of some unexpected responses to a molar constraint. We refer to Experi- 
ment 3 , in which the rats performed more extra licks and licked more efficiently as the price of water increasedsensible adaptations by the rat viewed as an optimal forager.

One final aspect of Experiment 3 merits special attention-the negative linear relation between total leverpresses and total licks. Similar experiments have demonstrated bitonic functions between the size of the instrumental requirement and the total amount of instrumental responding. Pertinent to our study, Allison and Boulter (1982, Experiment 3) showed that as the instrumental requirement increased, the total amount of instrumental leverpressing for water first increased, then decreased. Economically, these results imply that demand for water at low behavioral prices was inelastic, and at high behavioral prices, elastic (Allison, 1983). In contrast, our study demonstrated inelastic demand for water even at the highest prices.

Perhaps demand is inelastic under some conditions, but elastic under others. For example, Hursh (1986) suggested that demand for an essential commodity may be relatively inelastic in a relatively "closed" economy, one that offers few substitutes for the commodity in question. Perhaps studies that have demonstrated bitonic functions (e.g., Kelsey \& Allison, 1976) have used economies more "open" than those used in studies that have demonstrated linearity (e.g., Allison et al., 1979; Allison \& Moore, 1985). Several studies, however, appear to contradict this hypothesis: These studies have demonstrated both linear and bitonic functions in the same setting, with no manipulation of the extent to which the external economy is open or closed (e.g., Teitelbaum, 1957).

Whatever the source of the difference might be, our results underscore an important implication, which is generally overlooked, for "instrumental bitonic" settings. These are experimental settings that consistently reveal a bitonic relation between the size of the instrumental requirement and the total amount of instrumental responding. Recall that we found no significant difference between our instrumental and contingent series. Accordingly, we would expect an instrumental bitonic setting to be able to reveal a bitonic relation between the size of the contingent reward and the total amount of instrumental responding. In other words, if instrumental responding first rises and then falls as the instrumental requirement decreases, one should be able to reproduce the same bitonic function by increasing the magnitude of contingent reward.

Some studies have shown one or the other part of that bitonic reward function. Studies that have shown the bottom part, in which instrumental responding increases with magnitude of reward, include a recent example in which humans received pay to withstand pain (Cabanac, 1986).
Studies that have shown the top part, in which instrumental responding decreases as magnitude of reward increases, include our Experiment 3. (For a review of other studies that have shown the top part of the function, see Allison et al., 1979; for another recent example, see Collier, Johnson, Hill, and Kaufman, 1986.)

Numerous molar models imply the bitonic relation between instrumental responding and magnitude of reward, including the minimum deviation model (Staddon, 1979). Accordingly, we are surprised to have seen no experimental demonstration of the complete bitonic reward function. As our own results showed no difference between the instrumental and contingent series, they imply that both bitonic functions should be discoverable in any setting that typically reveals either one of the two.

\section{REFERENCES}

Allison, J. (1976). Contrast, induction, facilitation, suppression, and conservation. Journal of the Experimental Analysis of Behavior, 25 , 185-198.

Allison, J. (1983). Behavioral economics. New York: Praeger.

Allison, J., \& Boulter, P. (1982). Wage rate, nonlabor income, and labor supply in rats. Learning \& Motivation, 13, 324-342.

Allison, J., Miller, M., \& Wozny, M. (1979). Conservation in behavior. Joumal of Experimental Psychology: General, 108, 4-34.

Allison, J., \& MoOre, K. E. (1985). Lick-trading by rats: On the substitutability of dry, water, and saccharin tubes. Journal of the Experimental Analysis of Behavior, 43, 195-213.

Allison, J., Timberlake, W. (1974). Instrumental and contingent saccharin licking in rats: Response deprivation and reinforcement. Learning \& Motivation, 5, 231-247.

Cabanac, M. (1986). Money versus pain: Experimental study of a conflict in humans. Journal of the Experimental Analysis of Behavior, 46, $37-44$.

Collier, G. H., Johnson, D. F., Hill, W. L., Kaufman, L. W. (1986). The economics of the law of effect. Journal of the Experimental Analysis of Behavior, 46, 113-136.

Ferster, C. B., \& SinNer, B. F. (1957). Schedules of reinforcement. New York: Appleton-Century-Crofts.

Gleitman, H. (1983). Basic psychology. New York: Norton.

Hursh, S. R. (1986, June). Factors defining open and closed economies: Mapping the continuum. In M. L. Commons (Chair), Ninth Harvard symposium on quantitative analyses of behavior. Cambridge, MA.

Kelsey, J. E., \& Al.uson, J. (1976). Fixed-ratio lever pressing by VMH rats: Work vs. accessibility of sucrose reward. Physiology \& Behavior, 17, 749-754.

Staddon, J. E. R. (1979). Operant behavior as adaptation to constraint. Joumal of Experimental Psychology: General, 108, 49-67.

Teitelbaum, P. (1957). Random and food-directed activity in hyperphagic and normal rats. Joumal of Comparative \& Physiological Psychology, 50, 486-490.

Timberlake, W., \& Aluson, J. (1974). Response deprivation: An empirical approach to instrumental performance. Psychological Review, 81, 146-164.

(Manuscript received October 15, 1986; revision accepted for publication May 1, 1987.) 